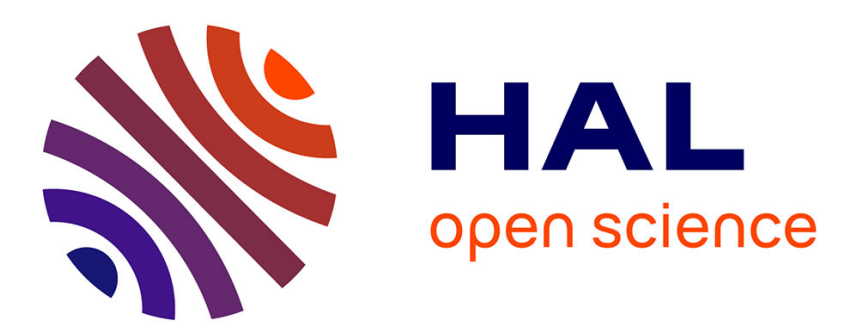

\title{
Oscillator Frequency Stability Improvement by Means of Negative Feedback
}

\author{
Maxim Goryachev, Serge Galliou, Philippe Abbe, Vadim Komine
}

\section{To cite this version:}

Maxim Goryachev, Serge Galliou, Philippe Abbe, Vadim Komine. Oscillator Frequency Stability Improvement by Means of Negative Feedback. IEEE Transactions on Ultrasonics, Ferroelectrics and Frequency Control, 2011, 58 (11), pp.2297-2304. 10.1109/TUFFC.2011.2087 . hal-00660546

\section{HAL Id: hal-00660546 \\ https://hal.science/hal-00660546}

Submitted on 13 Apr 2021

HAL is a multi-disciplinary open access archive for the deposit and dissemination of scientific research documents, whether they are published or not. The documents may come from teaching and research institutions in France or abroad, or from public or private research centers.
L'archive ouverte pluridisciplinaire HAL, est destinée au dépôt et à la diffusion de documents scientifiques de niveau recherche, publiés ou non, émanant des établissements d'enseignement et de recherche français ou étrangers, des laboratoires publics ou privés. 


\title{
Oscillator Frequency Stability Improvement by Means of Negative Feedback
}

\author{
Maxim Goryachev, Serge Galliou, Philippe Abbé, and Vadim Komine
}

\begin{abstract}
A novel, simple method is proposed to increase the frequency stability of an oscillator. An additional negative feedback is used in combination with the positive loop of the harmonic oscillator to decrease the phase sensitivity to fluctuations of parameters other than the resonator. The main advantage of the proposed correction approach is that it does not require expensive external elements such as mixers or resonators. The validity of the method is theoretically demonstrated on a Colpitts oscillator using the control system theory approach and numerical simulations, and is experimentally verified with phase noise measurements of an actual oscillatormockup. It is shown that the medium-term frequency stability can be easily improved by a factor of ten.
\end{abstract}

\section{INTRODUCTION}

$I^{x}$ NCREASING the oscillator frequency stability [1]-[3] is an important issue for many modern systems dealing with communication, navigation, radar applications, etc. Traditionally, frequency stability is achieved by minimizing the environmental influence, maximizing the loaded quality-factor $Q$ of the resonator, minimizing the noise of the sustaining amplifier, and optimizing the oscillator elements. Even if the oscillator phase noise performance is optimized by these means, it can be further improved by other noise reduction techniques. A summary of the most common amplifier and oscillator feedforward and feedback noise reduction techniques is given in [4]. Some of these techniques are VCO-based locking involving one of the following principles: high- $Q$ resonance element as an external discriminator [5], Pound discriminator [6]-[8], or carrier suppression using a high- $Q$ cavity [9]. The main disadvantage of such techniques is that an additional resonant element is required, which is usually the most critical device and adds its own noise. To avoid this, at least two other techniques may be considered: interferometric carrier suppression [10]-[12], and the high- $Q$ cavity as an external discriminator [13], [14]. These techniques use the information extracted from the oscillating loop for its correction with the voltage-controlled phase shifter. When analyzing all of these approaches, one common idea can be retained: all of the techniques try to transform the oscillator phase noise into a dc voltage by a phase detec-

M. Goryachev, S. Galliou, and Philippe Abbé are with the Time and Frequency Department of the FEMTO-ST Institute, Besançon, France (e-mail: maxim.goryachev@femto-st.fr).

V. Komine is with the Department of Electronics and Microprocessor Systems of the Ivanovo State Power University, Ivanovo, Russia. tion for further feedback correction. In contrast with these traditional phase-noise reduction approaches [4], the idea developed in this paper does not require a signal-phaseto-voltage conversion. The proposed structure decreases the dependency of the oscillation frequency on fluctuations of oscillator components other than the resonator. This improvement is achieved because of the high-level improvement of the oscillator architecture (structural optimization) and not by a parametric optimization as is proposed, for example, in [15].

A basic harmonic oscillator consists of a resonator and a sustaining amplifier interconnected in the form of a positive-feedback closed loop [16]. To sustain harmonic oscillations, the loop must be designed with respect to the well-known Barkhausen conditions. In actual applications, both the amplifier and the resonator are subject to parametric fluctuations. Because the oscillator feedback is positive, these fluctuations reproduce themselves after passing through the loop, according to the Leeson effect [17]. This results in a multiplication of the close-to-carrier open loop phase-noise power spectral density (PSD) by $f^{-2}$ within the resonator bandwidth. To avoid this drawback, the sign of the feedback must be negative as it is in a phase-locked loop (PLL) [18]. Indeed, the resulting PSD of a PLL depends on the open-loop PSD of its voltagecontrolled oscillator (VCO). Unfortunately, the PLL requires an external frequency reference source because a single negative loop is not able to sustain oscillations. As a result, the output close-to-carrier noise depends on the noise of this external frequency source. In addition, at least two resonators are used in this case to implement the system: one is in the VCO and another in the external reference. Is it possible to combine the ability of the usual positive feedback to sustain the oscillation and the property of the negative feedback to reduce the close-to-carrier phase noise by means of their structural integration using just one resonator? In this paper we try to answer this question by combining both in one oscillator.

It must be noted that the proposed method of phasenoise reduction is not an automatic gain control (AGC). The proposed solution deals with the reduction of oscillator frequency, but not amplitude fluctuations. Thus, the amplitude detector and a traditional variable gain amplifier are not used (even though detectorless AGC systems can also be of great interest). Instead, the oscillating signal is fed back into a sustaining amplifier with no averaging or detection of any kind.

The system under investigation is based on a common Colpitts quartz-crystal oscillator. The corresponding analytical description [19] differs from traditional approaches 


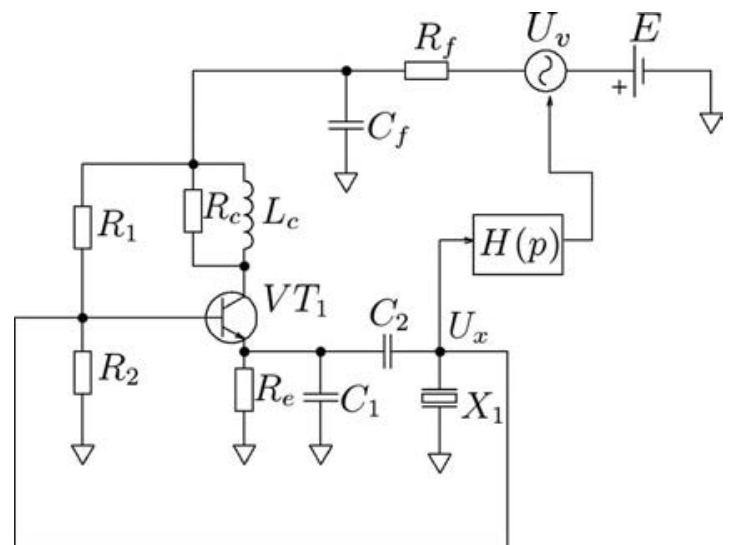

Fig. 1. Modified Colpitts oscillator with the second negative feedback.

[20]-[22]. Also, for simplicity, some elements are assumed to be ideal. The analytical results are further confirmed with numerical models using a commercial circuit simulator. This is an intermediate stage toward a conceptual verification. Though an actual implementation of the analyzed circuit is obviously slightly more complicated, both analytical and simulation investigations are in good qualitative agreement with experimental results. The improvement of the oscillator long-term frequency stability is demonstrated.

\section{MODEl DEScRiption}

The analyzed crystal oscillator is shown in Fig. 1. This modified Colpitts design has its usual positive feedback loop including the crystal filter and a sustaining amplifier. An additional negative feedback includes an idealized transfer function $H(p)$ (where $p \equiv \mathrm{d} / \mathrm{d} t$ is the differentiation operator) which drives a voltage-controlled voltage source $U_{\mathrm{v}}$. The first feedback loop is intended to sustain oscillations whose frequency is mainly defined by the crystal $(X 1)$ resonance frequency, slightly shifted by other oscillator components such as $C_{1}$ and $C_{2}$. The second feedback loop uses the voltage across the resonator to reinject the oscillating signal into the sustaining amplifier, but with an open-loop phase shift close to $\pm \pi$.

The main idea of the proposed analysis is to simplify the design through successive equivalent transformations $(\Delta-$ $Y$ and $Y-\Delta$ transforms, series and parallel connections, transforms of dependent signal sources) and to investigate the influence of the second negative feedback loop [different solutions for $H(p)$ ]. The oscillator is analyzed like a feedback system, as is usual in the control system theory.

At the first step, the oscillator must be properly transformed. In addition to equivalent transformations of the linear elements, the transistor is represented with its wellknown Ebers-Moll model. The result is shown in Fig. 2. This oscillator representation has only one nonlinear element: the base-emitter junction modeled with $I_{\mathrm{e}}\left(V_{\mathrm{be}}\right)$. Special attention should be paid to the fact that elements $Z_{\mathrm{x}}, Z_{1}$, and $Z_{\mathrm{e}}$ are functions of the parameters $C_{1}, R_{\mathrm{e}}, C_{2}$, and $X_{1}$, or more precisely,

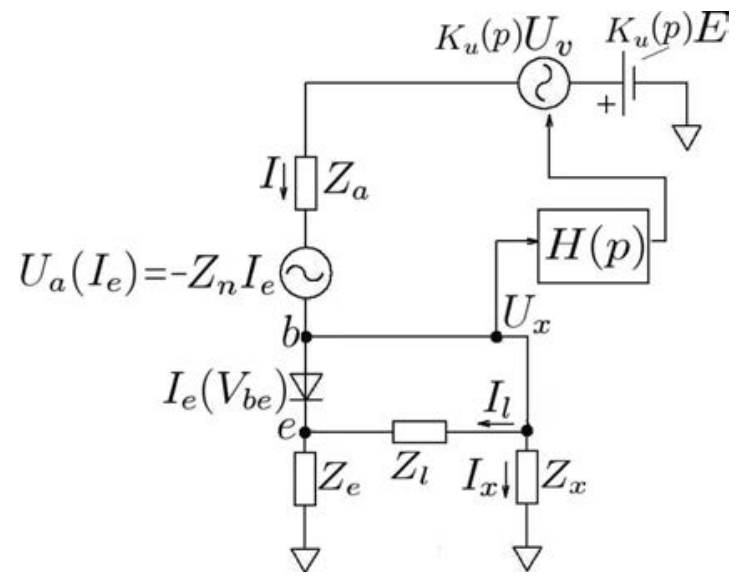

Fig. 2. Transformed two-loop Colpitts oscillator.

$$
Z_{\mathrm{x}}=p L_{\mathrm{x}}+\frac{1}{p C_{\mathrm{x}}}+R_{\mathrm{x}}, \quad Z_{\mathrm{l}}=\frac{1}{p C_{2}}, \quad Z_{\mathrm{e}}=\frac{1}{p C_{1}} \| R_{\mathrm{e}}
$$

where $L_{\mathrm{x}}, C_{\mathrm{x}}$, and $R_{\mathrm{x}}$ are the motional parameters of the crystal resonator. Other elements of the system in Fig. 2 do not depend on $C_{1}, R_{\mathrm{e}}, C_{2}$, and $X_{1}$. The voltagedependent element $K_{\mathrm{u}}(p) U_{\mathrm{v}}$ is the result of an equivalent transformation of resistor $R_{\mathrm{f}}$, the ideal voltage-controlled voltage source $U_{\mathrm{v}}=H(p) U_{\mathrm{x}}$ and the dc voltage source $E$ parallel to the filtering capacitor $C_{\mathrm{f}}$. Thus, $K_{\mathrm{u}}(p)$ is a transfer function depending on the resistor and the capacitor.

Using Kirchoff laws, the following system of equations may be written to describe the oscillator shown in Fig. 2, taking into account that the ideal block $H(p)$ has no input current:

$$
\left\{\begin{array}{l}
\quad K_{\mathrm{u}}(p) E+U_{\mathrm{x}} K_{\mathrm{u}}(p) H(p)+I_{\mathrm{e}} Z_{\mathrm{n}}=I Z_{\mathrm{a}}+U_{\mathrm{be}} \\
\quad+\left(I_{1}+I_{\mathrm{e}}\right) Z_{\mathrm{e}}, \\
I=I_{\mathrm{e}}+I_{\mathrm{l}}+I_{\mathrm{x}}, \\
U_{\mathrm{x}}=V_{\mathrm{be}}+\left(I_{1}+I_{\mathrm{e}}\right) Z_{\mathrm{e}}, \\
I_{\mathrm{e}}\left(U_{\mathrm{be}}\right)=I_{\mathrm{s}} \exp \left(\frac{V_{\mathrm{be}}}{u_{\mathrm{t}}}-1\right),
\end{array}\right.
$$

where $I$ is a current through elements $E_{\mathrm{u}}, U_{\mathrm{a}}\left(I_{\mathrm{e}}\right)=-Z_{\mathrm{n}}(p)$ $I_{\mathrm{e}} . I_{\mathrm{S}}$ and $u_{\mathrm{t}}$ are transistor parameters.

System (2) corresponds to the idealized physical system shown in Fig. 2. The same system may be represented from the point of view of control system theory, i.e., using blocks of transfer functions as follows (see Fig. 3):

$$
\begin{aligned}
K_{\mathrm{a}} & =1+\frac{Z_{\mathrm{e}}}{Z_{1}}, \\
K_{\mathrm{s}} & =\frac{Z_{\mathrm{x}} Z_{1}}{Z_{\mathrm{x}} Z_{1}+Z_{\mathrm{e}} Z_{\mathrm{x}}+Z_{\mathrm{a}} Z_{\mathrm{e}}+Z_{\mathrm{a}} Z_{1}+Z_{\mathrm{a}} Z_{\mathrm{x}}}, \\
Z_{\mathrm{fb} 1} & =Z_{1} \frac{\left(Z_{\mathrm{a}}-Z_{\mathrm{n}}\right) Z_{\mathrm{x}}+Z_{\mathrm{a}} Z_{\mathrm{e}}+Z_{\mathrm{e}} Z_{\mathrm{x}}}{Z_{\mathrm{x}} Z_{1}+Z_{\mathrm{e}} Z_{\mathrm{x}}+Z_{\mathrm{a}} Z_{\mathrm{e}}+Z_{\mathrm{a}} Z_{1}+Z_{\mathrm{a}} Z_{\mathrm{x}}} .
\end{aligned}
$$




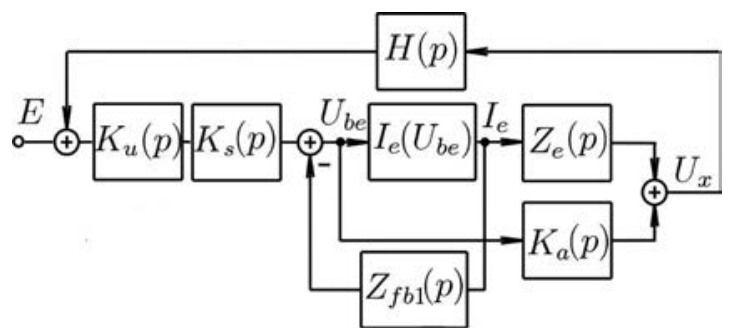

Fig. 3. Block-diagram representation of the Colpitts oscillator with the additional feedback [a path with the transfer functions $Z_{\mathrm{e}}(p), K_{\mathrm{a}}(p)$, and $H(p)]$.

In the same way as an original circuit, the system shown in Fig. 3 represents a two-loop nonlinear system with positive and negative feedbacks. The nonlinearity is represented with a nonlinear relationship $I_{\mathrm{e}}\left(U_{\mathrm{be}}\right)$. The common positive loop is represented with the linear transfer function (or physically the impedance) $Z_{\mathrm{fb} 1}(p)$ and the nonlinear transconductance $G=I_{\mathrm{e}}\left(U_{\mathrm{be}}\right)$. The negative loop consists of the linear transfer functions $K_{\mathrm{a}}(p), H(p)$, $K_{\mathrm{s}}(p), Z_{\mathrm{a}}(p), K_{\mathrm{u}}(p)$, and the nonlinear transconductance $G=I_{\mathrm{e}}\left(U_{\mathrm{be}}\right)$.

It must be noted that if $H(p) \equiv 0$, the second loop is broken and the system dynamics are characterized only by the linear transfer function $Z_{\mathrm{fb} 1}(p)$, and the corresponding amplitude is determined by nonlinear transconductance $G$ and impedance $Z_{\mathrm{fb} 1}(p)$. This case corresponds to the classical Colpitts oscillator. If $H(p) \neq 0$, the two-loop system may be equivalently transformed into a system with one loop and another linear transfer function $Z_{\mathrm{fb} 2}(p)$.

Further, for simplicity, it is assumed that $K_{\mathrm{u}}(p)=1$ without lack of generality. This is possible when the capacitance $C_{\mathrm{f}}$ is large enough.

\section{Model ANALysis}

The nonlinear dynamic system shown in Fig. 3 can be represented by nonlinear and linear parts in one closed loop. Different methods to find oscillation amplitude and frequency are known for this type of nonlinear systems: for example, the method of describing functions [23], [24], also called the harmonic balance method in control theory. This method is very useful for high-order systems and helps to find the oscillation conditions from the open-loop transfer function of the whole system.

In the absence of the additional negative feedback, the linearized open-loop transfer function of the oscillator can be expressed as

$$
H_{\mathrm{ol} 1}=-G_{0} Z_{\mathrm{fb}},
$$

where $G_{0}$ is a linearized transconductance of the nonlinear element $I_{\mathrm{e}}\left(U_{\mathrm{be}}\right)$ in the vicinity of the operating point.

As previously mentioned, if the second loop exists, it may be represented as a modification of the first positive loop. In this case, one can also calculate the open-loop transfer function of the oscillating loop as

$$
H_{\mathrm{ol} 2}=-G_{0} Z_{1} \frac{\left(Z_{\mathrm{a}}-Z_{\mathrm{n}}\right) Z_{\mathrm{x}}+Z_{\mathrm{a}} Z_{\mathrm{e}}+Z_{\mathrm{e}} Z_{\mathrm{x}}(1-H(p))}{Z_{\mathrm{a}}\left(Z_{1}+Z_{\mathrm{e}}+Z_{\mathrm{x}}\right)+(1-H(p)) Z_{\mathrm{x}}\left(Z_{1}+Z_{\mathrm{e}}\right)},
$$

Thus, for example, in the ideal case of $H(p)=1$, the transfer function (5) is considerably simplified:

$$
H_{\mathrm{o} 12}=-G_{0} Z_{1} \frac{\left(1-\frac{Z_{\mathrm{n}}}{Z_{\mathrm{a}}}\right) Z_{\mathrm{x}}+Z_{\mathrm{e}}}{Z_{1}+Z_{\mathrm{e}}+Z_{\mathrm{x}}} .
$$

The existence of oscillations is found from Barkhausen conditions, which may be written as $\Im\left\{H_{\mathrm{ol} i}\right\}=0$ and $\left|H_{\mathrm{ol} i}\right|$ $>0$, where $i \in\{1,2\}$ and $\Im\{\}$ denotes an imaginary part. From the first equation of the Barkhausen conditions, one may write the equation necessary for calculating the oscillation frequency. Thus, in the absence of the second loop, the oscillating frequency may be found from the sixth-order equation

$$
\begin{aligned}
& \omega^{6}-\omega^{4}\left[2 \omega_{0}^{2}-\frac{\omega_{0}^{2}}{Q^{2}}+\omega_{1}^{2}+\omega_{\Sigma}^{2}-\frac{1}{\tau_{1} \tau_{\Sigma}}\right] \\
& +\omega^{2}\left[\omega_{0}^{4}+\frac{\omega_{0}^{2}}{Q^{2} \tau_{1} \tau_{\Sigma}}-\frac{2 \omega_{0}^{2}}{\tau_{1} \tau_{\Sigma}}+\omega_{0}^{2} \omega_{\Sigma}^{2}+\omega_{0}^{2} \omega_{1}^{2}\right. \\
& \left.+\omega_{1}^{2} \omega_{\Sigma}^{2}+\frac{2 \omega_{0} \omega_{\Sigma}}{\tau_{1} Q}\right]+\frac{\omega_{0}^{4}}{\tau_{1} \tau_{\Sigma}}=0,
\end{aligned}
$$

where

$$
\begin{aligned}
& \omega_{0}^{2}=\frac{1}{C_{\mathrm{x}} L_{\mathrm{x}}}, \quad Q=\frac{L_{\mathrm{x}} \omega_{0}}{C_{\mathrm{x}}}, \quad \omega_{1}^{2}=\frac{1}{K_{\mathrm{m}} C_{1} L_{\mathrm{x}}}, \\
& \omega_{\Sigma}^{2}=\frac{C_{1}+C_{2}}{L_{\mathrm{x}} C_{1} C_{2}}, \quad \tau_{1}=K_{\mathrm{m}} R_{\mathrm{m}} C_{1}, \quad \tau_{\Sigma}=R_{\mathrm{m}} \frac{C_{1} C_{2}}{C 1+C 2} .
\end{aligned}
$$

For simplicity, it is assumed that $R_{\mathrm{m}}=Z_{2}$ and $K_{\mathrm{m}}=$ $1-Z_{\mathrm{n}} / Z_{\mathrm{a}}$ are real. This is relevant when the presence of $L_{\mathrm{c}}$ may be neglected. In this work all of the parameters defined in (8) are considered fluctuating with time.

For the case of a system having a second loop with a transfer function $H(p)=1$, the oscillating frequency is determined by the fourth-order equation

$$
\begin{aligned}
& \omega^{4}-\omega^{2}\left[2 \omega_{0}^{2}-\frac{\omega_{0}^{2}}{Q^{2}}+\omega_{1}^{2}+\omega_{\Sigma}^{2}\right]+\omega_{0}^{4}+\omega_{0}^{2}\left(\omega_{1}^{2}+\omega_{\Sigma}^{2}\right) \\
& \quad+\omega_{\Sigma}^{2} \omega_{1}^{2}=0,
\end{aligned}
$$

with two physically based solutions in the case of a high quality factor $Q: \omega^{2} \approx \omega_{0}^{2}+\omega_{1}^{2}$ and $\omega^{2} \approx \omega_{0}^{2}+\omega_{\Sigma}^{2}$.

For the further analysis, this case of high- $Q$ resonators is considered, i.e., that of crystal resonators whose $Q \cdot f_{0}$ product is greater than $1 \cdot 10^{13}$ ( $Q$ is typically close to $1 \cdot 10^{6}$ at $\left.f_{0}=\omega_{0} /(2 \pi)=10 \mathrm{MHz}\right)$. Then, all the terms 
inversely proportional to $Q$ can be neglected in (7) and (9). In fact, (7) and (9) are of the same type, and can be represented in the following form:

$$
\omega^{6}-\omega^{4}\left[\alpha_{1}+q \beta_{1}\right]+\omega^{2}\left[\alpha_{2}+q \beta_{2}\right]+q \beta_{3}=0,
$$

where $q$ is a generic parameter, such that if $q=0,(10)$ reduces to (9), and if $q=1$, this equation represents the system without correction, i.e., described by $(7): \beta_{1}=-$ $\left(1 /\left(\tau_{1} \tau_{\Sigma}\right)\right), \beta_{2}=-\left(2 \omega_{0}^{2}\right) /\left(\tau_{1} \tau_{\Sigma}\right), \beta_{3}=\left(\omega_{0}^{4}\right) /\left(\tau_{1} \tau_{\Sigma}\right)$. Consequently,

$$
\begin{aligned}
& \alpha_{1}=2 \omega_{0}^{2}+\omega_{1}^{2}+\omega_{\Sigma}^{2}, \\
& \alpha_{2}=\omega_{0}^{4}+\omega_{0}^{2}\left(\omega_{1}^{2}+\omega_{\Sigma}^{2}\right)+\omega_{\Sigma}^{2} \omega_{1}^{2} .
\end{aligned}
$$

To compare the frequency sensitivity in both cases, small changes of parameters around their mean values must be considered. Thus, the oscillating frequency is a sum of a constant value $\omega_{\mathrm{c}}$ and a small change $\Delta \omega$. The same representation can be made for equation parameters, i.e., $\alpha_{1}=\alpha_{1 \mathrm{c}}+\Delta \alpha_{1}, \alpha_{2}=\alpha_{2 \mathrm{c}}+\Delta \alpha_{2}, \beta_{1}=\beta_{1 \mathrm{c}}+\Delta \beta_{1}, \beta_{2}$ $=\beta_{2 \mathrm{c}}+\Delta \beta_{2}, \beta_{3}=\beta_{3 \mathrm{c}}+\Delta \beta_{3}$. Next, substituting these expressions into (10) and neglecting the higher-order terms of small variations, an expression for the frequency change can be deduced:

$$
\begin{aligned}
\Delta \omega= & \frac{\omega_{\mathrm{c}}^{4} \Delta \alpha_{1}-\omega_{\mathrm{c}}^{2} \Delta \alpha_{2}}{6 \omega_{\mathrm{c}}^{5}-4 \omega_{\mathrm{c}}^{3}\left(\alpha_{1 \mathrm{c}}+q \beta_{1 \mathrm{c}}\right)+2 \omega_{\mathrm{c}}\left(\alpha_{2 \mathrm{c}}+q \beta_{2 \mathrm{c}}\right)} \\
& +q \frac{\omega_{\mathrm{c}}^{4} \Delta \beta_{1}-\omega_{\mathrm{c}}^{2} \Delta \beta_{2}-\Delta \beta_{3}}{6 \omega_{\mathrm{c}}^{5}-4 \omega_{\mathrm{c}}^{3}\left(\alpha_{1 \mathrm{c}}+q \beta_{1 \mathrm{c}}\right)+2 \omega_{\mathrm{c}}\left(\alpha_{2 \mathrm{c}}+q \beta_{2 \mathrm{c}}\right)}
\end{aligned}
$$

Eq. (12) clearly shows that in the case of $q=0$, the last term does not exist. It can also be easily shown that the denominator of both terms slightly depends on $q$ in the case $\omega_{\mathrm{c}}^{2}-\omega_{0}^{2} \ll \omega_{0}^{2}$, which is also relevant. It can be noted that the second term could vanish even for $q=1$ in the marginal case $\omega_{\mathrm{c}}=\omega_{0}$. In this case, the oscillating frequency depends only on $\omega_{0}$ and no improvement is achieved with the second feedback. However, $\omega_{\mathrm{c}}$ never actually equals $\omega_{0}$ (although the condition $\omega_{\mathrm{c}}^{2}-\omega_{0}^{2} \ll \omega_{0}^{2}$ holds true), simply because some other reactive components exist in the circuit. Moreover, resonators are intentionally fabricated with a resonant frequency less than the desired oscillation frequency. The difference is usually tuned with a series capacitance (not shown in our schematics, for simplicity) in the course of oscillator design. This is done to cover resonator parameter dispersion. So, in reality, the second term in (12) always exists for $q=1$. Thus, the circuit sensitivity to parameter fluctuations can be decreased. The improvement effect exists even for parameters $L_{\mathrm{x}}$ and $C_{\mathrm{x}}$ of the resonator itself (again only in the case $\left.\omega_{\mathrm{c}} \neq \omega_{0}\right)$. Thus, this analysis demonstrates that the oscillator with the second feedback is less sensitive to parametric fluctuations, because its related frequency change $\Delta \omega$ has fewer contributing terms of instability.

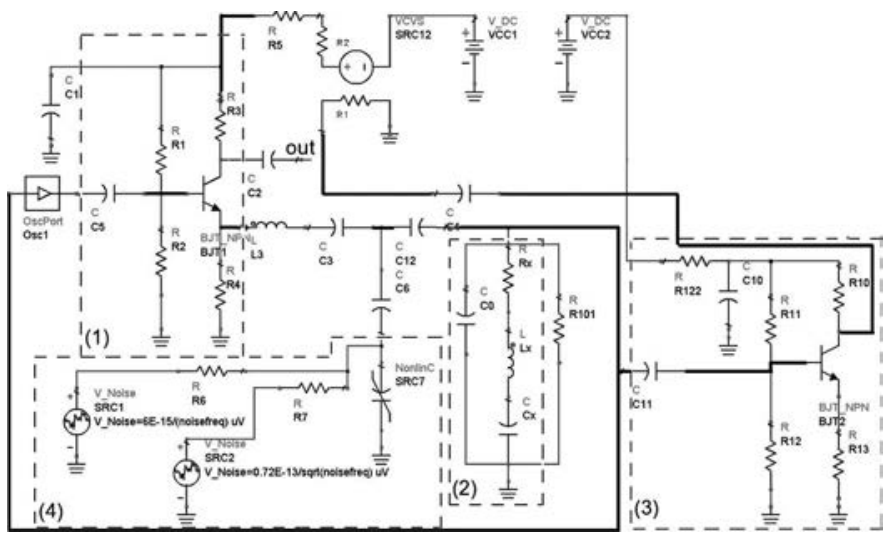

Fig. 4. Advanced Design System (ADS) design of the two-loop system: (1) sustaining amplifier, (2) crystal resonator, (3) buffer amplifier, (4) parametric noise source. Signal paths of both loops are shown in bold.

In general, the resonator should always be considered as a BAW device in its fluctuating environment (electrical, thermal, mechanical). Therefore, the proposed method can be considered as an improvement of the electrical environment in terms of the oscillating frequency's sensitivity to circuit component fluctuations.

The sign of the transfer function $H(p)=1$ is justified by the fact that the second feedback is inherently negative because of the sign introduced by the system configuration. In the same way, the first loop is positive in spite of the sign in (4)-(6). In the case of a practical circuit, in which the sustaining amplifier of the oscillating loop is more complicated, the negative sign of the second feedback is introduced by an inverting amplifier.

\section{System Simulation}

To verify the results of the previous section, the phase noise of the considered oscillator has been simulated with Advanced Design System (ADS, Agilent Technologies Inc., Santa Clara, CA) (for phase noise simulation principles see, for example, [3], [25]). An ADS design of the system is shown in Fig. 4. This design represents a more realistic variant of the circuit shown in Fig. 1, with an additional common-emitter amplifier in the oscillator negative feedback. The operational concept remains the same. The voltage across the crystal resonator is amplified and injected back into the sustaining amplifier.

During the oscillator phase-noise simulation, special attention is devoted to the parametric noise of circuit components, because these component instabilities are supposed to be suppressed by the second loop. Because the usual commercial circuit simulators do not contain any parametric noise models, we solve this problem with the approach that has been successfully used for precision close-to-carrier phase-noise simulations [26]. To introduce parametric phase fluctuations into the system, a parametric noise source is implemented in the circuit (see Fig. 4). This source includes a voltage-controlled capacitor SRC7, 


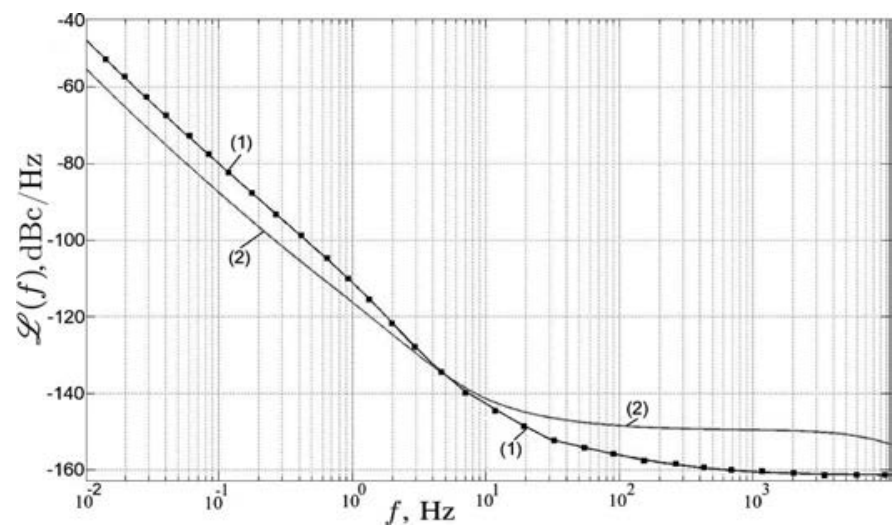

Fig. 5. Simulated effect of the oscillator negative feedback on the oscillator phase-noise power spectral density [single-sideband noise spectrum $\mathcal{L}(f)$ : (1) one-loop oscillator, (2) two-loop oscillator.

whose value depends on the voltage of two noise sources SRC1 and SRC2. Indeed, voltage noise is converted into parametric capacitance change, and further into oscillation frequency fluctuations related to oscillator phase noise. Thus, the first source SRC1 must have a PSD proportional to $f^{-1 / 2}$ to produce an open-loop $f^{-1}$ noise for medium and high Fourier frequencies, because the created noise on SRC7 has a spectral density proportional to the squared voltage of the noise source SRC1. In other words, SRC1 is a source of parametric flicker noise. Similarly, the second source SRC2, with a PSD proportional to $f^{-1}$, gives an $f^{-2}$ slope of the open-loop noise for very low frequencies (which is mainly caused by temperature randomwalk fluctuations, physically). In other words, SRC2 is a source of parametric random walk. These noise sources are calibrated to be dominant for low Fourier frequencies.

Fig. 5 shows the simulation results in terms of $\mathcal{L}(f)$, the phase-noise PSD, of the oscillator with and without the second feedback. The PSD of the oscillator without the second feedback [see curve (1), Fig. 5] for Fourier frequencies lower than $5 \mathrm{~Hz}$, i.e., inside the resonator bandwidth, is dominated by the parametric noise introduced as described previously. This conclusion is made by comparing the results with and without the parametric noise source. The slopes of these curves close to the carrier are due to the Leeson effect [17] applied to the parametric phase noise. Usually $f^{-2}$ (because parametric random walk is dominant for $f<5 \cdot 10^{-2} \mathrm{~Hz}$ ) and $f^{-1}$ (because parametric flicker is dominant for $5 \cdot 10^{-2}<f<2 \mathrm{~Hz}$ ) are observed here. For the higher frequencies, the oscillator phase noise is mainly due to the additive noise of its components. The second feedback considerably reduces $f^{-3}$ parametric noise for low Fourier frequencies by about $9 \mathrm{dBc} / \mathrm{Hz}$ and almost eliminates $f^{-4}$ instabilities for the very low Fourier frequencies (curve (2), Fig. 5). These effects strongly correlate with the amplification and phase shift in the second system feedback. Also it must be noted that in the absence of the parametric noise source, the effect of the additional feedback is negligible in the close-tocarrier region. Therefore, when comparing both curves, it can be seen that the frequency stabilization effect exists

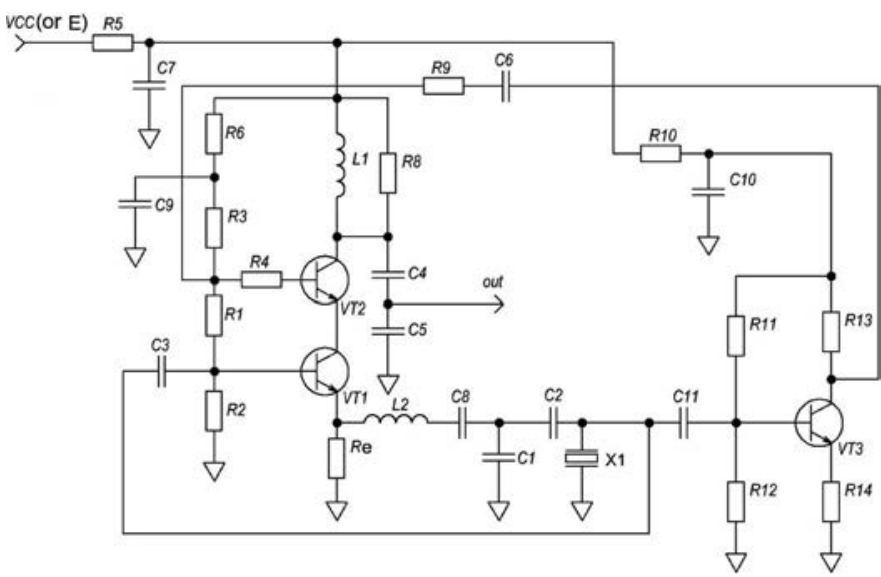

Fig. 6. Practical realization of the two-loop oscillator. L2 and C8 are added to reject resonator spurious modes.

for parametric instabilities in the region of low Fourier frequencies, where parametric fluctuations are dominant. For higher offset frequencies, the second feedback increases instabilities because of the additional noise of the amplifier in the second feedback. The level of this additive noise is mostly determined by the signal-to-noise ratio in the second feedback.

Thus, the simulation results confirm the analytical analysis made in the previous section. The second negative feedback reduces the oscillator frequency sensitivity to fluctuations of its parameters inside the resonator bandwidth despite an increase outside this bandwidth. Using the same simulation techniques, the effectiveness of this approach has also been confirmed for another crystal oscillator topology known as a Pierce oscillator.

\section{EXPERIMENTAL VERIFICATION}

A $10-\mathrm{MHz}$ quartz crystal oscillator with two feedback loops has been designed (see Fig. 6) to verify the actual efficiency of the expected phase-noise correction. The implemented mockup-oscillator is based on a cascode amplifier stage with two independent inputs. One input (base of transistor VT1) is connected directly with the resonator and locks the oscillating loop. The other input (base of transistor VT2) is connected to the same resonator, but through the common-emitter amplifier based on transistor VT3, which shifts the phase (ideally) by $\pi$ to introduce the appropriate correction to the oscillating loop. Though this circuit is much more complicated than the one described in the previous sections, it works on the same principles. The actual circuit parameters are chosen to meet the assumptions made in the previous sections. Particularly, the input current of the inverting amplifier is much less than that of the sustaining one to meet the assumption of a high input impedance in $H(p)$.

The circuit operation has been tested in two configurations to find out the effect of the additional negative feedback. In the first operation mode [see Fig. 7, curves (1) 


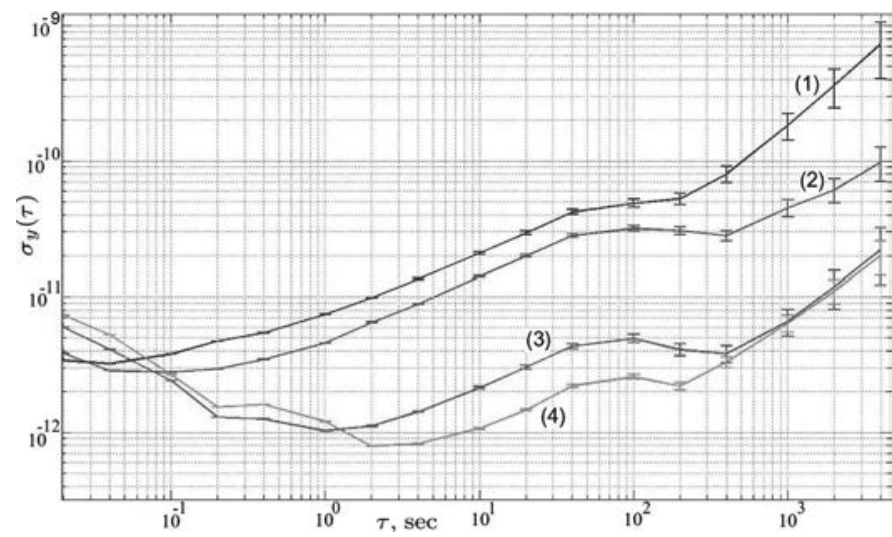

Fig. 7. Effect of the oscillator negative feedback on the Allan deviation: (1) 18-V supply voltage, one-loop oscillator, (2) 18-V supply voltage, two-loop oscillator, (3) 5-V supply voltage, one-loop oscillator, (4) 5 -V supply voltage, two-loop oscillator.

and (3)], this second feedback is unlocked. Thus, for example, resistor R9 is substituted with an open circuit. So, the feedback inverting amplifier is present in the circuit, but it is not locked to the sustaining amplifier. After that, the same circuit is tested with small values of this resistor, i.e., in operation with both loops [see Fig. 7, curves (2) and (4)]. The experiment is repeated for several values of the supply voltage VCC.

The goal of this design is not to built an ultra-stable oscillator (that necessarily requires a complex and expensive thermo-mechanical structure), but to demonstrate the efficiency of the phase-correction loop. The whole circuit is simply placed inside a Dewar flask with just a single temperature control of the resonator case. Moreover, the circuit lacks output matching amplifiers and is not parametrically optimized.
Measurements have been made with a TSC 5120A phase noise test set (Timing Solutions Corp., Boulder, $\mathrm{CO}$ ) using a hydrogen maser as a reference.

The results in Fig. 7 show that for all values of the supply voltage, the long-term frequency stability of the oscillator is improved. At the same time, its short-term stability is slightly degraded because of additional noise of the transistor VT3 in the second feedback. Even though the transistor VT1 is saturated in the $18-\mathrm{V}$ configuration, the effect of stability improvement exists. In the $5-\mathrm{V}$ experiment, this transistor is in the linear operating mode, and in this case the oscillator exhibits better results than for $18 \mathrm{~V}$. Thus, when nonlinearities are very limited, the negative feedback loop is still efficient. This fact confirms the linear nature of the experimentally observed effect. The effect of the negative feedback is also seen on the corresponding phase-noise PSDs which are shown in Fig. 8. This effect is qualitatively the same as predicted by the simulation (see Fig. 5).

The experimental results are in good agreement with simulation data. Parts of the plots on the left of the minima are determined by the white phase noise (see Fig. 5) and mostly depend on the signal-to-noise ratio. In the case of $18-\mathrm{V}$ power supply, better performances are achieved in this region. Here also, the second feedback degrades the system frequency stability. This fact corresponds to the increase of white phase noise for high Fourier frequencies in Fig. 5. Next, the minima of the Allan deviation are determined by the oscillator flicker frequency noise, i.e., the $f^{-3}$ phase noise in Fig. 5 . Here, the second feedback results in stability improvement with a simultaneous shift of the minima toward the higher $\tau$. Frequency stability improvement is clearly observed for the intermediate values of $\tau$ (up to about $200 \mathrm{sec}$ ) where the Allan

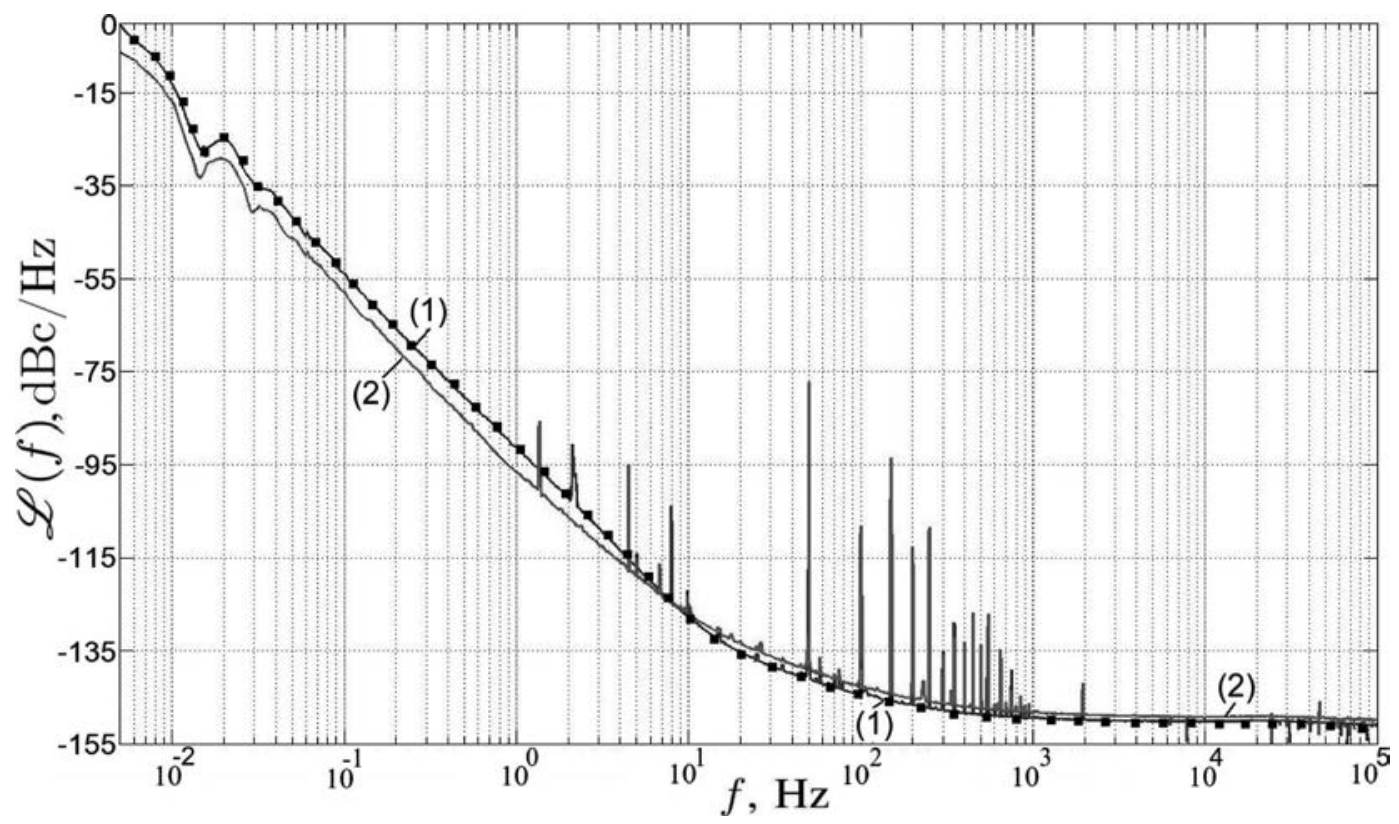

Fig. 8. Effect of the oscillator negative feedback on the phase-noise power spectral density [single-sideband noise spectrum $\mathcal{L}(f)$ ]: $(1)$ one-loop oscillator, (2) two-loop oscillator. 
deviation corresponds to $f^{-3}$ and $f^{-4}$ phase noise $\left(\tau^{0}\right.$ and $\tau^{+1 / 2}$ slopes, respectively). This effect has been predicted by the simulation. In addition, the effect of the second feedback decreases for very long averaging times $\tau$ in the $5-\mathrm{V}$ case. Indeed, the very-long-term frequency stability of such oscillators mainly originates from resonator parametric instabilities and aging (all of the sources of oscillator frequency instabilities are still discussed [27]-[29]) and, thus, according to the suggested analytical analysis, it can hardly be improved. These effects were not simulated previously. In the $18-\mathrm{V}$ case, the improvement still exists, meaning that these instabilities originate from other sources not previously considered here.

\section{Conclusions}

In this work, investigations of the oscillator architecture are presented. The introduction of a negative feedback loop was suggested in addition to the positive one that sustains the oscillation. The second additional feedback reinjects the inverted signal from the crystal resonator into the sustaining amplifier. It is shown analytically, using a simple model, that this second feedback is able to make the oscillating frequency less dependent on the circuit parameters, and therefore less sensitive to the parametric fluctuations of the circuit. The same idea is further demonstrated with numerical simulations. Additionally, the simulation analysis states that for high Fourier frequencies, the oscillator phase noise could be increased. These conclusions are also confirmed with actual frequency stability measurements of a mock-up oscillator. A non-negligible frequency stability improvement is observed in the area of middle and high averaging times, $\tau$. On the other hand, the short-term frequency stability is slightly degraded because of additive noise of the additional electronic components. These results are achievable for medium-quality oscillators with no additional costs on high-quality resonators, mixers, or temperature control units, which are the most expensive components. The phase-correction loop could be an alternative solution for a crystal oscillator positioned between the temperature-compensated crystal oscillator (TCXO) type and the oven-controlled crystal oscillator (OCXO) type [30]. In further work, the same principle of frequency stability improvement should be investigated for state-ofthe-art oscillators.

\section{ACKNOWLEDGMENTS}

The authors thank the anonymous reviewers for their invaluable comments and suggestions.

\section{REFERENCES}

[1] J. Rutman, "Characterization of phase and frequency instabilities in precision frequency sources: Fifteen years of progress," Proc. IEEE, vol. 66, pp. 1048-1075, Sep. 1978.
[2] V. F. Kroupa, Ed. Frequency Stability: Fundamentals and Measurement. New York, NY: IEEE Press, 1983.

[3] A. Demir, A. Mehrotra, and J. Roychowdhury, "Phase noise in oscillators: A unifying theory and numerical methods for characterization," IEEE Trans. Circuits Syst. I, vol. 47, pp. 655-674, May 2000.

[4] C. McNeilage, E. A. Ivanov, P. R. Stockwell, and J. H. Searls, "Review of feedback and feedforward noise reduction techniques," in Proc. IEEE Int. Frequency Control Symp., 1998, pp. 146-155.

[5] F. L. Walls, C. M. Felton, and T. D. Martin, "High spectral purity x-band source," in Proc. IEEE Symp. Frequency Control, 1990, pp 542-547.

[6] R. V. Pound, "Electronic frequency stabilization of microwave oscillators," Rev. Sci. Instrum., vol. 17, no. 11, pp. 490-505, 1946.

[7] A. N. Luiten, A. G. Mann, M. E. Costa, and D. G. Blair, "Cryogenic sapphire resonator oscillator with exceptional frequency stability: An update," in Proc. IEEE Int. Frequency Control Symp., 1994, pp. 441-446.

[8] D. G. Santiago, R. T. Wang, and G. J. Dick, "Improved performance of a temperature compensated LN2 cooled sapphire oscillator," in Proc. IEEE Int. Frequency Control Symp., 1995, pp. 397-400.

[9] D. G. Santiago and G. J. Dick, "Microwave frequency discriminator with a cryogenic sapphire resonator for ultra-low phase noise," in Proc. IEEE Frequency Control Symp., 1992, pp. 176-182.

[10] E. N. Ivanov, M. E. Tobar, and R. A. Woode, "Tunable microwave oscillator for low phase noise applications," in Proc. IEEE Int. Frequency Control Symp., 1997, pp. 985-993.

[11] E. N. Ivanov, M. E. Tobar, and R. A. Woode, "Advanced phase noise suppression technique for next generation of ultra-low noise microwave oscillators," in Proc. IEEE Int. Frequency Control Symp., pp. 314-320, 1995.

[12] E. N. Ivanov, M. E. Tobar, and R. A. Woode, "Ultra-low noise microwave oscillator with advanced phase noise suppression system," IEEE Microw. Guid. Wave Lett., vol. 6, no. 9, pp. 312-314, 1996.

[13] D. P. Tsarapkin and V. S. Komarov, "Stable microwave oscillator with combined stabilization," Proc. Moscow Power Engineering Inst., vol. 151, no. 51, pp. 82-86, 1973.

[14] Z. Galani, M. J. Bianchini, R. C. Waterman, R. Dibiase, R. W. Laton, and J. Bradord Cole, "Analysis and design of a single-resonator GaAsFET oscillator with noise degeneration," Trans. IEEE Microw. Theory Tech., vol. 32, no. 12, pp. 1556-1565, 1984.

[15] F. Filicori and G. Vannini, "Frequency stability in resonator-stabilized oscillators," IEEE Trans. Circ. Syst., vol. 37, no. 11, pp. 1440-1444, Nov. 1990.

[16] E. Rubiola, Phase Noise and Frequency Stability in Oscillators. New York, NY: Cambridge University Press, 2008.

[17] D. B. Leeson, "A simple model of feedback oscillator noise spectrum," Proc. IEEE, vol. 54, no. 2, pp. 329-330, 1966.

[18] J. Klapper and J. T. Frankle, Phase-Locked and Frequency-Feedback Systems. New York, NY: Academic, 1972.

[19] M. Goryachev, S. Galliou, and Ph. Abbé, "Oscillator phase noise optimization and correction," in Proc. European Frequency and Time Forum, Noordwijk, The Netherlands, 2010.

[20] R. W. Rhea, Oscillator Design and Computer Simulation. 2nd ed. Atlanta, GA: Noble Publishing, 1995.

[21] B. Parzen, Design of Crystal and Other Harmonic Oscillators. New York, NY: Wiley, 1983.

[22] R. Quere, J. C. Nallatamby, A. Layec, and M. Prigent, "Low phase noise oscillator modeling in a system environment," IEEE Int. Microwave Symp. Dig., 2005, vol. 2, pp. 891-894.

[23] W. S. Levine, Control System Fundamentals. New York, NY: CRC Press, 1999.

[24] D. P. Atherton, Stability of Nonlinear Systems, New York, NY: Wiley, 1981.

[25] T. Djurhuus, V. Krozer, J. Vidkjaer, and T. K. Johansen, "Oscillator phase noise: A geometrical approach," IEEE Trans. Circuits Syst. I, vol. 56, no. 7, pp. 1373-1382, Jul. 2009.

[26] M. Goryachev and S. Galliou, "Precision close-to-carrier phase noise simulation of BAW oscillators," IEEE Trans. Ultrason. Ferroelectr. Freq. Control, vol. 58, no. 1, pp. 6-9, Jan. 2011.

[27] F. L. Walls, "The quest to understand and reduce $1 /$ f noise in amplifiers and BAW quartz oscillators," in Proc. European Frequency and Time Forum, Besançon, France, Mar. 8-10, 1995, pp. 227-243.

[28] R. Brendel, M. Olivier, and G. Marianneau, "Analysis of the internal noise of quartz-crystal oscillators," IEEE Trans. Instrum. Meas., vol. 22 , pp. 160-170, Jun. 1975.

[29] E. Rubiola and V. Giordano, "On the $1 /$ f frequency noise in ultrastable quartz oscillators," IEEE Trans. Ultrason. Ferroelectr. Freq. Control, vol. 54, no. 1, pp. 15-22, Jan. 2007.

[30] J. R. Vig, Quartz crystal resonators and oscillators: For frequency control and timing applications - A tutorial, 2004. 